\title{
Actuación Resiliente durante un Desastre con Equipos de Actuación Inmediata
}

\section{Resilient action during a disaster with Immediate Reaction Teams}

\author{
Mayor de Mg. Ricardo Javier Acuña López \\ Academia de Guerra de la Fuerza Terrestre \\ rjacunal@ejercito.mil.ec
}

\begin{abstract}
Resumen
Las amenazas de la naturaleza a través de los tiempos han tenido graves consecuencias irreparables, a causa de catástrofes que han evidenciado su poder destructivo. El impacto social, económico y estructural ha afectado a países como Colombia, Chile, China, Ecuador, Haití, Indonesia y Perú. Esto ha originado una serie de preocupaciones en entidades internacionales como las Naciones Unidas, que ha planteado mecanismos que permitan mitigar los efectos, generando iniciativas integrales con los actores gubernamentales en cada uno de los países, permitiendo gestiones para perfeccionar su resiliencia. Las experiencias del pasado conducen a que Ecuador determine la importancia de proponer la participación de instituciones y equipos entrenados para actuar en las primeras 24 horas luego del siniestro. Ante este escenario, este estudio tiene como objetivo determinar una estructura ideal para la participación de equipos que actúen de manera inmediata en coordinación con las instituciones del Estado ecuatoriano. Para lograrlo, se desarrolló una investigación bibliográfica mediante un exhaustivo análisis de varias fuentes primarias y secundarias, a más de experiencias similares derivadas en el campo de la gestión de riesgos de países de la región. Además, se realizó un análisis de la normativa que rigen la Gestión de Riesgos de Desastres en el Ecuador. Los resultados revelan el diseño de una organización que permita mantener un grupo homogéneo con jerarquización, misiones y tareas específicas a la reacción en desastres naturales o antrópicos preparados para su actuación temprana.
\end{abstract}

Palabras clave: Desastre Natural, Emergencia, Prevención, Resiliencia, Salvamento.

\begin{abstract}
Through times, the natural hazards have marked severe and irreparable consequences due to catastrophes that have been evidence of their destructive powers. The social, economic, and structural impact has affected Colombia, Chile, China, Ecuador, Haiti, Indonesia, and Peru. Thus, this has raised a series of concerns for international entities such as the United Nations, which has proposed mechanisms to mitigate the effects, generating comprehensive initiatives with government actors in each country, allowing efforts to improve their resilience. Based on this background, the current study aims to determine an ideal structure for the participation of teams that act immediately in coordination with the institutions of the Ecuadorian State. The present research developed a bibliographic search through an exhaustive analysis of several primary and secondary sources towards more than similar experiences derived in risk management in countries of the local region. The experiences from past conduct Ecuador to determine the importance of creating institutions and specialized teams trained to act in the first 24 hours after the adverse has occurred any event. In addition, this study analyzed the regulations governing Disaster Risk Management in Ecuador. The results reveal the design of an organization, which allows maintaining a homogeneous group with hierarchy, missions, and specific tasks to the reaction in natural or anthropic disasters trained for its early action.
\end{abstract}

Keywords: Natural Disaster, Emergency, Prevention, Resilience, Rescue. 


\section{Introducción}

En los últimos años, las emergencias, catástrofes y desastres han estado presentes en toda Latinoamérica, tales como: terremotos, aluviones e incendios forestales que, como consecuencia del cambio climático, ya son parte de un acontecer reiterado. En otras palabras, los riesgos existentes se amplificarán con estos fenómenos constantes del calentamiento global, lo que es un tema que preocupa a muchos organismos.Así por ejemplo, Puyeta (2017) señala que: "Los estudios han demostrado que este hecho es consecuencia directa del cambio climático y que, de no tomar medidas, la situación empeorará en el futuro."

Todo parece confirmar que los incendios forestales, tormentas eléctricas, olas de calor y frío, inundaciones, ciclones y huracanes aparecerán de una manera común y su predicción es compleja. Más aún que los desastres están "aumentando en frecuencia e intensidad, que obstaculizan significativamente el progreso al desarrollo sostenible." (Del Castillo, 2015)

Ante estos hechos, el trabajo de las Fuerzas Armadas ha sido específico por su participación de manera fundamental en la preparación, reacción y reconstrucción, siempre dentro de los protocolos que han normado de cierta manera su actuación, como parte de un sistema estatal para la reducción del riesgo y desastres, ajustados a las reglas internacionales previstas para tal efecto.

Ante este escenario, este estudio tiene como objetivo determinar una estructura ideal para la participación de equipos que actúen de manera inmediata en coordinación con las instituciones del Estado ecuatoriano. Para lograrlo, se desarrolló una investigación bibliográfica, mediante un exhaustivo análisis de varias fuentes primarias y secundarias a más de experiencias similares derivadas en el campo de la gestión de riesgos de países de la región. Además, se realizó un análisis de la normativa que rige la Gestión de Riesgos de Desastres en el Ecuador. Así mismo, se propone la importancia de la participación de varios estamentos gubernamentales para generar una resiliencia con gobernanza de manera efectiva y precisa.

Por otro lado, se estructura un planteamiento ideal que permita a las Fuerzas Armadas actuar desde una perspectiva de reacción enmarcada en las capacidades de actuación inmediata de acuerdo a su organización, jurisdicción y equipamiento, de conformidad con la misión constitucional y las consideradas en la política de defensa en referencia a la gestión de desastres.

Finalmente, todas las fuentes consultadas en su mayoría han sido bibliográficas, con un sustento que permita tener una perspectiva que, en forma general, aporta definiciones del accionar que vinculan el trabajo de diversas instituciones de seguridad para actuar de manera temprana ante los sucesos que generen los riesgos naturales o antrópicos.

La principal contribución de este estudio es presentar de una manera conceptual la estructura idónea de una organización doctrinaria, los equipos de reacción inmediata, que en coordinación con el ejército determinarían las acciones de ayuda durante las primeras 24 horas de ocurrido los hechos.

El resto del artículo ha sido estructurado como sigue: en primer momento, se explica qué es la gestión de reducción de desastres. En un segundo, se desarrolla la concepción de la importancia de actuación en desastres en los países de la región. En el tercero, se analiza la importancia de preparar y entrenar grupos especializados de actuación inmediata. Finalmente, se realiza una analogía de lo que presenta el Ejército Ecuatoriano y la propuesta de una estructura acorde al empleo en el tema de desastres. 


\section{Materiales y Métodos}

\subsection{La Reducción de Riesgos y Desastres}

Del 18 a 22 de enero del año 2005, la Organización de las Naciones Unidas llevó a cabo la Conferencia Mundial sobre la Reducción de los Desastres, en Japón, cuya finalidad fue la de consolidar estrategias internacionales para la reducción de Desastres bajo la denominación del Marco de Acción de Hyogo ${ }^{1}$, con expectativas por alcanzar hasta el 2015. En esta conferencia se plantearon además tres objetivos estratégicos y cinco prioridades para aumentar la resiliencia de las naciones y las comunidades ante los desastres, que buscan como resultado final, reducir de una manera considerable toda clase de pérdidas, manteniendo como preferencia la vida del ser humano.

Así mismo, el 18 de marzo del 2015, a petición de la Asamblea General del Ecuador, se realizó la tercera conferencia mundial de las Naciones Unidas para la reducción de riesgos y desastres. Aquí se aprobaron las prioridades de acción dentro del Marco de Senday ${ }^{2}$ que, en su parte medular, plantean las acciones para reducir las amenazas y vulnerabilidades, ratificando el aumento de la resiliencia, fortaleciendo de esta forma el Marco de Acción de Hyogo. En sí, el propósito de este es el de colocar como actor principal y responsable directo a cada uno de los estados, lo que permite de esta manera la actuación de todas las sociedades e instituciones que lo conforman. Esto se enmarca en 4 prioridades, que han sido operacionalizadas con las gestiones de cada país. Esto, a su vez, también ha permitido que del apoyo en situaciones de desastres surjan actores con responsabilidades y tareas institucionalizadas dentro de la gestión para un desastre.

En concordancia con esto, han aparecido normativas y legalidades para la participación del personal uniformado como institución, para que se pueda actuar de manera organizada y en apoyo coordinado, lo cual permite que su misión vaya más allá de la protección de la frontera y los límites territoriales. En definitiva, el tema del salvamento, prevención y actuación inmediata (a nivel local, regional y mundial) ha demostrado en estos mismos momentos que, en base a experiencias pasadas, se trata de una preocupación con aspiraciones excepcionales, con expectativas para enfrentar la furia de la naturaleza cuando esta se anuncie. Esta preocupación conlleva a que desde varias aristas y actores se propongan estrategias dentro de un marco de la legalidad, para hacer efectivo el accionar expedito de las Fuerzas de Seguridad en momentos de mucha conmoción, que es la diferencia entre salvar vidas o recuperar cadáveres.

\subsection{Actuación de grupos de acción inmediata en desastres}

Mantener una reacción de forma apropiada y de manera ágil en eventos de daños naturales o socio naturales es la inquietud de todos los países a nivel internacional. Especialmente en aquellos que han enfrentado varias devastaciones de tipo económico y social, que dentro de un proceso de resiliencia han creado cifras incalculables que llevan a deliberar las acciones que deben realizarse desde la prevención.

España, con la intención de ponerse a la vanguardia en prevención y seguridad en el contorno de desastres, ha proyectado la creación de un grupo especializado que entra en acción de forma inmediata en la intervención de desastres, conformado por miembros de sus Fuerzas Armadas, bajo la denominación de Unidad Militar de Emergencia (UME), que a través del Acuerdo de Ministros del Consejo del 7 de Octubre del $2005^{3}$ crea una legislatura con todos sus protocolos, para que, a través de un mando unificado, su organización pueda actuar de manera eficaz en todo tipo de eventos. Estas

EIRD, Naciones Unidas. Conferencia Mundial sobre la Reducción de Desastres. Marco de Acción de Hyogo del 2005-2015. Japón: s.n., 2005.

ONU, Naciones Unidas. Marco de Senday para la Reducción del Riesgo de Desastres 2015-2030. Japón: s.n., 2015.

JAUREGUI A. Boletín Oficial del Estado. Madrid, España: s.n., julio de 2011. 178. 
unidades pretenden crear los elementos necesarios que permitan una reacción en el menor tiempo posible. Es por esto que, desde sus escuelas de formación en rescates, cumplen una estructura curricular que hace posible capacitar a todos sus miembros en los ramos necesarios para cubrir los escenarios que puedan presentarse.

La actuación de las UME ha mantenido su éxito por la conciencia que todos los actores del sistema de seguridad que apuntalan al mismo objetivo; desde las mismas Fuerzas Armadas, que visionan la preservación de la seguridad y el bienestar de sus ciudadanos. Así mismo, las entidades del estado destinan los recursos inexcusables para un despliegue de las fuerzas, tanto en el territorio nacional como internacional. Prueba de esto es su participación en la lucha contra los incendios de manera local en casi todo el verano, contribución de apoyo en los incendios que han asolado Chile, el actuar en Portugal y los terremotos en Nepal y Ecuador, todas estas de gran admiración y reconocimiento.

Elementos similares presenta Colombia con la Unidad Nacional para la Gestión del Riesgo de Desastres (UNGRD)4, que une a varios actores que ejecutan tareas cuyo actuar principal es la atención a la mitigación, prevención y operación en desastres, logrando la sincronización en la búsqueda y rescate. Cada uno de ellos está entrenado en sus particularidades y es de gran apoyo para sobrellevar escenas donde la zozobra es el factor primordial que debe vencerse. Así pues, ésta organización la conforman: la Unidad de Búsqueda y Rescate Col.1 (USAR Col.1)5 del cuerpo de bomberos, que ha tenido participación internacional en labores de búsqueda tanto en Haití, Ecuador y México y la Unidad de Operaciones Especiales en Emergencias y Desastres de la Policía Nacional (PONALSAR)6, cuyo accionar principal es el salvamento en el contorno urbano como esencia de su misión institucional. Por otro lado, el Ejército, Armada y Fuerza Aérea Colombiana, a través de un Batallón de Prevención y Atención a Desastres, cada uno con recursos y capital humano, son parte de este componente, para intervenir en el apoyo a la población.

Ecuador, por su parte, se encuentra en proceso de formación de las Unidades de Intervención Rápida (UNIR)7, en cumplimiento con la estructura del Sistema Nacional de Gestión de Riesgos, cuya necesidad aparece con las lecciones aprendidas de los acontecimientos del terremoto del 2016. Esta conformación considera en particular entrenar un número estimado de 170 hombres, equivalente a dos compañías por División, con el objetivo de cumplir misiones de primera respuesta dentro del territorio nacional, en concordancia con las entidades de la Gestión de Riesgos. Esto permite mantener personal, material y equipo en excelentes condiciones para hacer frente a los desastres naturales o antrópicos, a fin de salvar la mayor cantidad de vidas humanas de manera oportuna condescendiendo con la población civil.

Chile, en este contexto, es un país que ha sido afectado por varios eventos donde la naturaleza ha puesto a prueba la operatividad de sus Fuerzas Armadas, que han respondido con gran eficacia en las misiones impuestas, actuando bajo el estado de excepción en apoyo a las catástrofes y desastres, al tener que realizar organizaciones especiales para estos eventos. Una de éstas es la Patrulla de Auxilio y Rescate Militar del Ejército (PARME), cuyas unidades son empleadas en ciertas jurisdicciones. Sin embargo, lo más notable de esta nueva estructura es la creación y

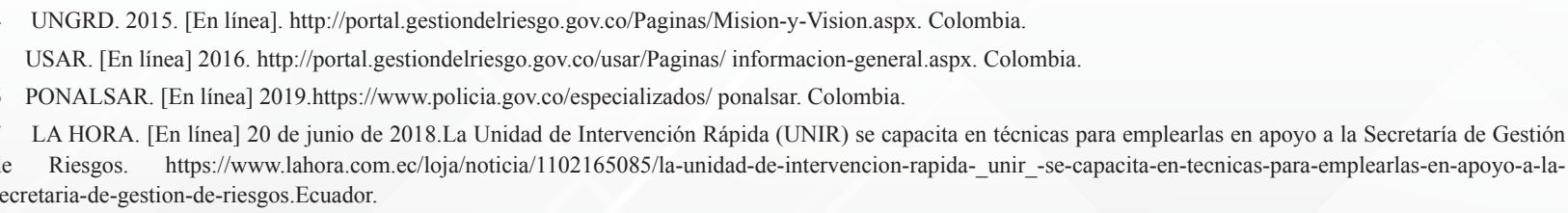


actuación de un personal altamente capacitado denominado BRIFE (Brigadas de Refuerzo de Incendios Forestales del Ejército), cuya formación y empleo está refrendado en una Resolución Exenta $\mathrm{N}^{\circ} 1011$ del 25 de noviembre de 2016, con excepcional reconocimiento por su desempeño en los incendios de febrero del 2017. Esto es resaltado por la Subsecretaría del interior en su informe final denominado "Tormenta de Fuego", en el que expresa:

“[...] las Brigadas de Refuerzo para Incendios Forestales del Ejército (BRIFE), prestan colaboración desde el principio en el combate del fuego en segundo anillo, después de los brigadistas $\mathrm{CONAF}^{8}$. Dentro de los ámbitos de colaboración se encuentra el de prevención y combate de incendios forestales. Una de las acciones permanentes $[\ldots]$ " 9

Por tanto, el tema de mantener equipos con material y conocimientos en apoyo a los desastres ya no es un tema de moda. La Cruz Roja Internacional siempre lo ha asumido en primer plano, puesto que mantiene en preparación constante un contingente operativo para actuar en cualquier momento, bajo la modalidad de formación de equipos nacionales de intervención en desastres conocidos como (ENI): "[...] los equipos son técnicamente preparados, especializados, equipados y actualizados para coordinar, facilitar y ejecutar acciones de respuesta, en beneficio de las personas más vulnerables. Sus funciones se enmarcan en la reducción, respuesta y recuperación de emergencias y desastres [...]" (Marroquín, 2015). Están compuestos por sistemas y medios combinados que les permiten tener respuesta nacional e internacional.

Lo dicho hasta aquí supone que las fuerzas del orden, y en sí las Fuerzas Armadas de todos los países, tienen la misión de proteger y salvar las vidas de sus ciudadanos en lo que a desastres se refiere. Más aún, la preparación para estas eventualidades requiere de la participación activa de muchos organismos, en la que resalta la actuación del servicio público, en especial de aquellos elementos que no están dentro los cuadros permanentes, generando así la necesidad y responsabilidad de empleo de manera organizada y especializada.

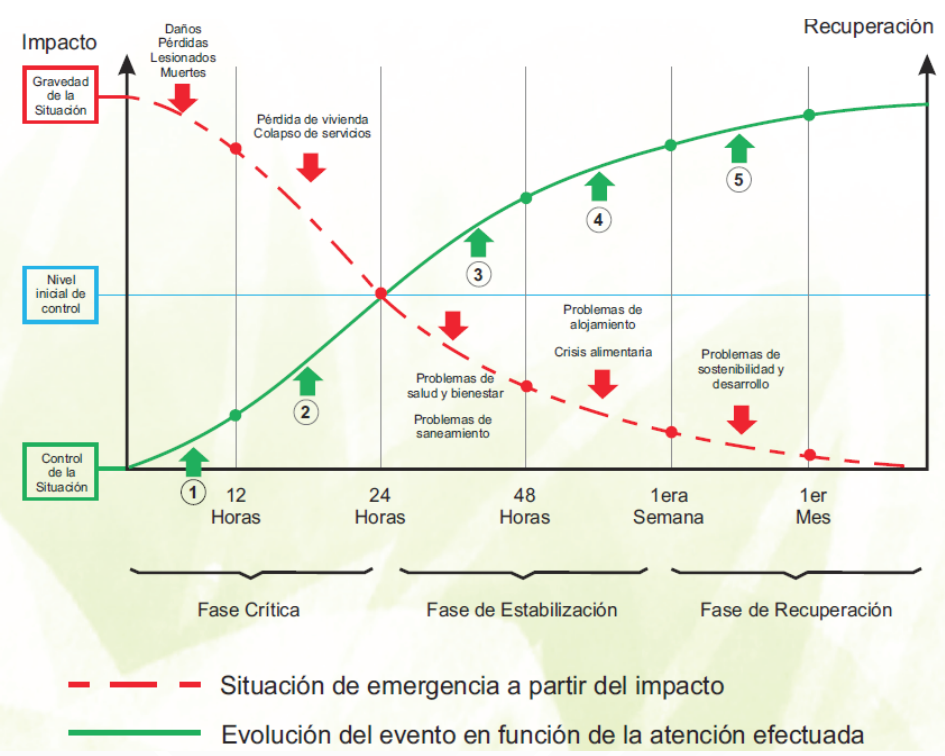

Figura 1: Fases de una situación general de desastre: propuesta descriptiva de SNPAD (2006). Fuente: La comunicación en la Gestión del Riesgo de Desastresv

8 CONAF: La Corporación Nacional Forestal es una institución autónoma del Estado chileno, creada originalmente como corporación de derecho privado, dependiente del Ministerio de Agricultura de Chile.

9 MINISTERIO DEL INTERIOR Y SEGURIDAD PÚBLICA. Chile y la tormenta de fuego. Informe Incendios Forestales. Chile: s.n., enero-febrero de 2017. pág. 41. 
Los miembros de las reservas, Fuerzas de resistencia, entre otras, poseen su participación activa en acciones de prevención, salvamento y también en apoyo a los más vulnerables, incluso en diversos acontecimientos. Esto exige formalizar una arista para precisar su actuar mediante una organización, legislatura y conocimientos que permitan desarrollar la resiliencia en cada uno de los eventos que puedan presentarse. De ahí que, es necesario que estas fuerzas, que al parecer pasan de improviso, cumplan una actuación directa, en especial en las fases 1 y 2 del impacto y evolución de los desastres (ver Figura 1), donde es impredecible que se realicen todas las acciones de salvamento iniciales y cooperación, para atender las víctimas directas del evento.

La Figura $\mathrm{N}^{\circ} 1$ muestra la evolución del evento, iniciando en una la fase crítica que requiere una acción inmediata en las primeras 24 horas de ocurrido el suceso. Aquí aparece una escalada del control de la situación y empieza un gran impacto por la gravedad de la situación durante el inicio del siniestro. Superadas las 24 horas, se llega a un nivel de control que permite mantener una fase de estabilización y llegar a la fase de recuperación, donde las acciones de control y gravidez son totalmente opuestas a las del inicio, esto se propone un tiempo mínimo de 30 días.

En el Ecuador, el terremoto del 16 de abril del 2016 puso a prueba a toda la organización de las Fuerzas Armadas para una atención prioritaria en los sectores más afectados. Las consecuencias de una organización distinta llevaron a considerar que la participación de personas externas permite una interrelación de mejora de procesos en medio de la zozobra y la desesperanza de las poblaciones afectadas. Contar con una organización afín a las tareas de actuación inmediata, habría beneficiado los procesos de resiliencia en apoyo a las entidades del Estado. Por ejemplo, habría sido útil durante la confección de 12000 raciones diarias, más de 150 misiones diarias de transporte de kits humanitarios y la entrega de víveres y vituallas, además de las operaciones de rescate en infraestructura colapsada y el soporte en la atención prioritaria en los más 200 albergues adecuados durante la emergencia.

\section{Evaluación de Resultados y Discusión}

\section{1. Evaluación de Resultados}

Muchos de los países han revelado que la reacción inmediata ya no es solo un paradigma, ya que el actuar de las fuerzas estatales es un hecho. En consecuencia, han visto necesario fortalecer un campo ocupacional para afrontar las vulnerabilidades que pudieran presentarse. Así, por ejemplo, en una investigación realizada en Colombia se acuerda ubicar a los Profesionales Oficiales de Reserva (POR) en actividades útiles para enfrentar desastres. Esta gestión es calificada como una estrategia acertada, ya que permite "diseñar propuestas ambiciosas y debidamente orientadas para emplear los POR en las tres instancias de gestión del riesgo, es decir, el conocimiento, la reducción y el manejo de desastres." (Herrera, 2018)

Algo semejante también propone el Dr. Marquín (2010) en Ecuador, bajo la creación de una Fuerza de Tarea que ayude a los sectores afectados, con capacidades y habilidades multidisciplinarias, enfatizando que esta organización: “[...] plantea el trabajo de grupo en el fomento de actividades de prevención, mitigación, reducción y respuesta en desastres para reducir la vulnerabilidad disminuir el riesgo en las comunidades (involucramiento y participación de la comunidad)." 
En esta misma línea, el Ejército Ecuatoriano determinó dentro de su doctrina "Equinoccio" la participación en apoyo a la gestión de riesgos. Se trata de una actuación de acuerdo a los niveles respuesta a través de la conformación de un contingente que permita una reacción de acuerdo a su jurisdicción, capacidad y periodo, formalizando de una u otra forma su actuar, su conformación, capacitación, equipamiento y su reacción inmediata de manera local y con proyección de reforzar zonas y regiones (ver Figura 2).

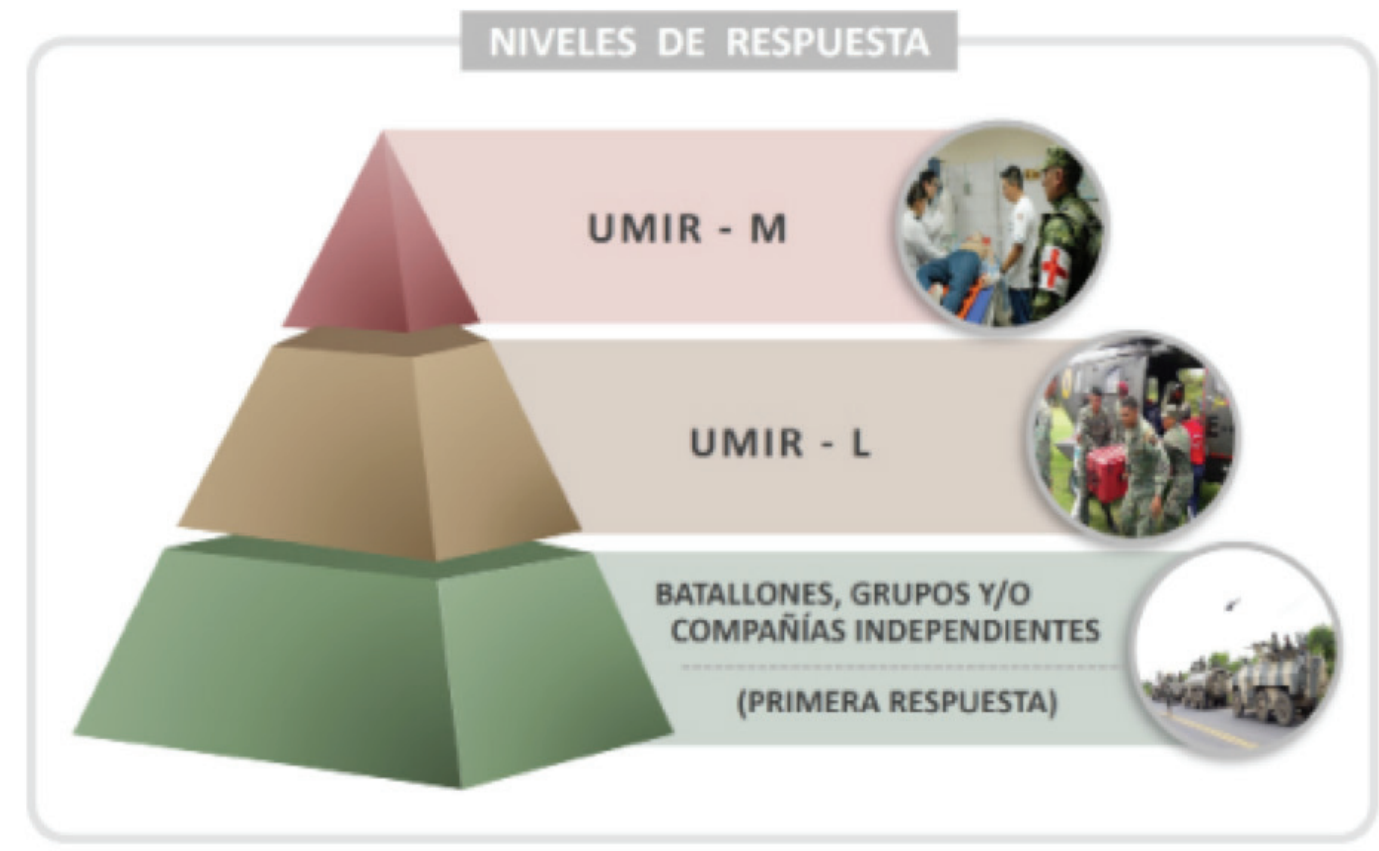

Figura 2: Niveles de respuesta en la gestión de riesgos (2021). Fuente: MFR. Apoyo a las instituciones del Estado

Como se puede observar, la Figura 2 ilustra una pirámide organizacional compuesta por tres niveles, en la que la primera respuesta está soportada por los Batallones y/o similares; posterior a esto, actúan las Unidades de intervención y rescate livianas con equipo para soportar hasta tres días de operación con un total de 20 hombres. Finalmente, sobre estas actúan las Unidades de intervención y rescate medianas con un contingente de 40 hombres, con todas las capacidades operativas y logísticas de soportar hasta 7 días de contingencia.

La participación de grupos previamente entrenados pretende que las acciones de resiliencia mejoren en la medida en que su actuación genere estabilidad al momento del desastre, lo cual disminuye la incertidumbre e introduce el momento propicio para aprovechar las competencias de todo el personal profesional existente en las diferentes ramas, de manera que este último pueda ser apoyo en varias tareas que se presenten, pues no todo es salvamento. Es así que se puede fortalecer las capacidades de un contingente patrón desde varios escenarios que permitan tomar la resiliencia de una mejor manera. Por ejemplo, en momentos de sosiego debe ser imperativa la participación de médicos, ingenieros, conductores, e incluso de efectivos que apoyan a la labor logística en la administración de las donaciones. Esto permite aprovechar todas las especialidades voluntarias pre-entrenadas, con el fin último de mantener una contribución activa durante todo el proceso que conlleva el actuar en una emergencia o desastre. 
A partir de todo el análisis bibliográfico presentado, es posible definir de una mejor perspectiva los roles del personal de reservistas y Fuerzas de resistencia, para que conformen unidades de participación y reacción inmediata, cuya intervención inicial debe primar desde los simulacros, de tal forma que se pueda perfeccionar un accionar con reentrenamientos en los que el fin común sea la atención a los conciudadanos mediante una participación efectiva, bajo el amparo del apoyo humanitario.

\section{2. Discusión}

Las amenazas inesperadas de la naturaleza han demostrado que lo primordial es la prevención a través de la preparación y el entrenamiento, para así mantener una actuación efectiva en el salvamento. De acuerdo a este estudio, es imperativa la propuesta de la participación del personal de reservistas y fuerzas de resistencia en acciones de resiliencia como acción inmediata, lo que permitiría mantener una actuación emblemática como parte del apoyo de las instituciones del Estado en el tema de desastres.

Es necesario para esto considerar que la participación de estas fuerzas debe mantener una organización bajo una estructura tipo Brigadas con los Centros de Reservistas y los cuarteles regionales de una manera anticipada. Por lo tanto, se determina imprescindible un levantamiento de las capacidades efectivas de cada uno de sus integrantes, según las especialidades, designando desde ya las responsabilidades específicas en el trabajo de apoyo a las emergencias, fundamentando que las prioridades "[...] versan sobre las vulnerabilidades de personas y subgrupos: niños, género, personas de edad, personas con discapacidad y apoyo psicosocial.”(Esfera, 2011)

Además, dada su ocupación, y por los objetivos que necesitan ser creados, este personal debe mantener una magnitud básica bajo la denominación de "Brigada" que les permita una actuación de manera inmediata en las regiones donde se encuentran acantonadas, y con las condiciones de actuar como refuerzos cerca de la circunscripción si ese fuera el caso. De esta forma, se mantendrá la capacidad de realizar operaciones de salvamento enmarcados en la legalidad, cumpliendo la misión de primera respuesta en capacidad de salvar la mayor cantidad de vidas humanas en el menor tiempo posible, obteniendo un efecto positivo en la sociedad asistida.

Bajo este contexto, se puede formar una estructura organizacional que permita mantener un grupo homogéneo con jerarquización, misiones y tareas específicas a la reacción en desastres naturales o antrópicos, apuntalados en tres equipos de especialidad para su actuación temprana (ver Figura 3).

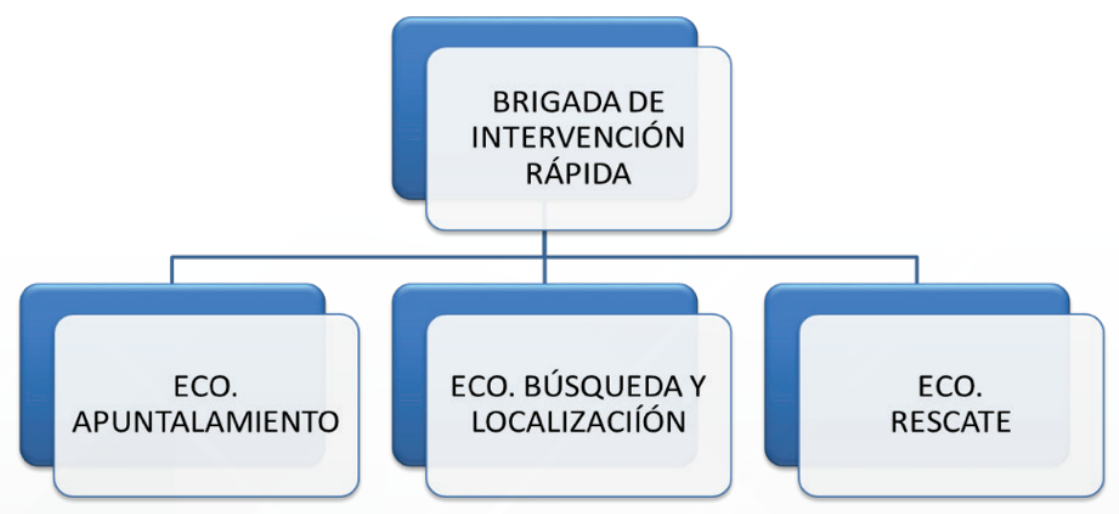

Figura 3: Propuesta de una Brigada con personal no profesional de FF.AA. Fuente: Ilustración propia 
La misión principal de esta unidad es crear las condiciones favorables de ayuda humanitaria de manera inmediata en cada una de las jurisdicciones con precisión y agilidad. Las brigadas deberán estar conformadas por un máximo de 12 hombres y comandadas por un profesional certificado en Gestión de Riesgos o con conocimientos vastos en el tema del manejo de recuperación ante un desastre. Esto permitirá la viabilidad del empleo de estos pequeños grupos de una manera práctica, cuyo perfil deberá incluir los siguientes conocimientos:

- Institucionalidad de la Cruz Roja Internacional y su empleo

- Legislación del sistema internacional para la reducción del riesgo del desastre

- El plan de emergencia para la reducción de riesgos de desastre

- La sincronización de la Seguridad y Defensa con la Gestión del riesgo y desastres.

En la actualidad, la tarea del apoyo a la Gestión de riesgos y desastres hace imperiosa la participación del personal de Reservistas y Fuerzas de resistencia. Para esto se debe mantener un entrenamiento constante, acorde a las nuevas exigencias y misiones confiadas a las Fuerzas Armadas en apoyo a los desastres. El Ecuador no debe ignorar el tema de mantener una Brigada de intervención inmediata con pequeños grupos que le permita un campo de actuación solvente, mediante un adiestramiento esencial cuyas prioridades deben enfocarse en lo siguiente:

- Organización y funciones de una unidad de intervención rápida con responsabilidades para equipos de búsqueda, rescate y seguridad

- Conocimiento del material y equipo que pudiesen ser empleados en las diferentes situaciones y tipos de emergencia y/o desastres

- Entrenamiento específico para apoyar en caso de: incendios forestales, erupciones volcánicas, deslaves, inundaciones, tsunamis y terremotos

- Procedimientos y protocolos por seguir en cada uno de los eventos

- Conocimiento de la normativa técnica y la sincronización con las instituciones que participan en la Gestión del desastre

- Conocimiento de medicina básica y primeros auxilios

Para este propósito, es necesario determinar un protocolo que permita una sincronización con las instituciones del Estado que se emplean a través del sistema de alarmas de emergencia y su relación estrecha con el Comando de operaciones de emergencia. Consecuentemente, esta propuesta de Brigada, al actuar de manera independiente, no deja de estar ajustada en el sistema de ayuda bajo la jerarquización de las Fuerzas Armadas, reconociendo que su accionar permitirá una ejecución de planes de contingencia necesarios al momento de la reacción.

Ahora bien, es imperioso, pese a la ambición que esto conlleva, que el actuar de este contingente tenga éxito, pues se deben, además, generar los recursos para que su logística sea sustentable en el empleo; hay que generar las condiciones de recursos para su equipamiento con material que 
esté ajustado a las eventualidades que puedan presentarse. Así mismo, es fundamental estructurar las órdenes de instrucción y entrenamiento necesarias que contengan todos los temarios indispensables para fortalecer su intervención.

\section{Conclusiones}

Esta investigación tuvo como propósito determinar una estructura organizacional para la participación de equipos que actúen de manera inmediata en coordinación con las instituciones del Estado ecuatoriano ante desastres naturales o antrópicos. Este estudio evidencia que las acciones para mejorar la resiliencia están a la orden del día y son una preocupación a nivel regional y mundial, determinadas dentro de un campo de acción que permite planificar y crear iniciativas para reaccionar de manera inmediata a las eventualidades. En consecuencia, resulta coherente y necesario el mantener efectivos con conocimientos y equipos que puedan solventar la ayuda de manera expedita, acorde a las exigencias internacionales enmarcadas en la ayuda humanitaria y el bienestar de la población.

De todo lo investigado y analizado, se hace impostergable la creación de un campo de acción para las fuerzas afines a la seguridad, como los miembros de las reservas y fuerzas de resistencia que actúen de manera inmediata con efectos deseables. Eso sí, con la salvedad que, para tal efectividad, es necesario que se encuentren bajo un mando unificado con capacidades multifacéticas y que proporcionen el apoyo inmediato a través de tareas esenciales, trabajos específicos y apoyo humanitario durante los desastres. Esto los hará capaces de salvar la mayor cantidad de vidas posible, sin entorpecer las operaciones de ayuda, ni mucho menos buscar un protagonismo de empleo en el área en que se desenvuelven las catástrofes. Así mismo, es fundamental que su organización preste una respuesta inmediata en apoyo a los Comandos Operativos de Emergencia, para intervenir de manera inmediata con un contingente capacitado y equipado adecuadamente, de rápida reacción, para así desvanecer la incertidumbre y mantener el optimismo en toda la población civil. Se debe, entonces, aprovechar el profesionalismo del efectivo disponible y empezar con el proyecto de capacitación, entrenamiento y equipamiento respectivo, para mantener una fuerza de soporte para los planes de contingencia en el empleo de las Fuerzas Armadas en el apoyo a la gestión de riesgos y desastres de manera ágil, óptima y efectiva.

\section{Referencias Bibliográficas}

C.E.D.M.T. Febrero de 2021. MFR. Apoyo a las instituciones del Estado. Doctrina EQUINOCCIO. .Ecuador.

DEL CASTILLO G. agosto de 2015. Centro de Estudios Estratégicos. Reseña del Marco de Acción Hyogo, su aplicación en Chile y su relación con la defensa. Chile: s.n.,

EIRD.2005. Conferencia Mundial sobre la Reducción de Desastres. Marco de Acción de Acción de Hyogo del 2005-2015. Japón: s.n.,

HERRERA G., VIVAS O. abril-junio de 2018.Gestión del riesgo y atención de desastres con Profesionales Oficiales de la Reserva del Ejército Colombiano. Revista Científica General José María Córdova. Colombia: ESMIC, Vol. 16, 22.

JAUREGUI A. julio de 2011. Boletín Oficial del Estado. Madrid, España. 
LA HORA. 20 de junio de 2018.La Unidad de Intervención Rápida (UNIR) se capacita en técnicas para emplearlas en apoyo a la Secretaría de Gestión de Riesgos. [En línea]. https://www.lahora.com.ec/ loja/noticia/1102165085/la-unidad-de-intervencion-rapida-_unir_-se-capacita-en-tecnicas-paraemplearlas-en-apoyo-a-la-secretaria-de-gestion-de-riesgos.

MALQUÍN V. noviembre de 2010.Propuesta para la formación de equipo de apoyo e intervención inmediata Task Force (Fuerza de Tarea) en Gestión de Riesgo y Multiamenaza. Quito. pág. 52.

MARROQUÍN T. 2015.Cruz roja Guatemalteca. Formación de equipos de intervención en desastres-ENI-. [En línea]. https://www.cruzroja.gt/noticias/formacion-de-equipos-nacionales-de-intervencion-endesastres-eni/.

MINISTERIO DEL INTERIOR Y SEGURIDAD PÚBLICA. enero-febrero de 2017. Chile y la tormenta de fuego. Informe Incendios Forestales. Chile. pág. 41.

ONU. 2015. Marco de Sendai para la Reducción del Riesgo de Desastres 2015-2030. Japón

PAYUETA E. diciembre de 2017.Futuro Sostenible. Así se relacionan los desastres naturales con el cambio climático. [En línea]. http://www.futurosostenible.elmundo.es/mitigacion/asi-se-relacionan-losdesastres-naturales-con-el-cambio-climatico.

PONALSAR. 2019. PONALSAR Colombia. [En línea]. https://www.policia.gov.co/especializados/ ponalsar.

ESFERA P. 2011.Carta humanitaria, y normas mínimas para respuesta humanitaria. Estados Unidos

RODRÍGUEZ A., TORRES S y HERNÁNDEZ A. 2013. La comunicación en la Gestión del Riesgo de Desastres: El papel de la relación comunidad y entorno. Colombia. pág. 63. ISBN: 978-958-878272-0.

UNGRD. 2015. http://portal.gestiondelriesgo.gov.co/Paginas/Mision-y-Vision.aspx.

USAR. 2016. http://portal.gestiondelriesgo.gov.co/usar/Paginas/informacion-general.aspx. Colombia. 